\title{
The reproductive lifespan of Onchocerca volvulus in West African savanna
}

\author{
A.P. Plaisier ${ }^{1}$, G.J. van Oortmarssen ${ }^{1}$, J. Remme ${ }^{2}$ and \\ J.D.F. Habbema ${ }^{1}$ \\ ${ }^{1}$ Department of Public Health and Social Medicine, Medical Faculty, Erasmus University Rotterdam \\ Rotterdam, The Netherlands, and ${ }^{2}$ Onchocerciasis Control Programme, Ouagadougou, Burkina Faso
}

(Received 8 February 1990; accepted 2 July 1990)

\begin{abstract}
The epidemiological model ONCHOSIM - a model and computer simulation program for the transmission and control of onchocerciasis - has been used to determine the range of plausible values for the reproductive lifespan of Onchocerca volvulus. Model predictions based on different lifespan quantifications were compared with the results of longitudinal skin-snip surveys undertaken in 4 reference villages during 13 to 14 years of successful vector control in the Onchocerciasis Control Programme in West Africa. Good fits between predicted and observed trends in skin microfilarial loads could be obtained for all villages. It is concluded that the reproductive lifespan of the savanna strain of $O$. volvulus lies between 9 and 11 years, and that $95 \%$ of the parasites reach the end of reproduction before the age of 13 to 14 years.
\end{abstract}

Key words: Onchocerca volvulus: Onchocerciasis; Parasite lifespan; Simulation model

\section{Introduction}

The productive lifespan of Onchocerca vulvulus is the major determinant of the required duration of onchocerciasis control operations which aim at interruption of transmission and the subsequent elimination of the local parasite reservoir (Roberts et al., 1967; Remme et al., 1986; Karam et al., 1987). Thus far, estimates for the average lifespan of the parasite and the variability around this average have been based on experimental infections in monkeys (Duke, 1980) and on cross-sectional nodulectomy surveys and skin-snip surveys in areas with different periods of vector control (Roberts et al., 1967; Karam et al., 1987). Based on extensive longitudinal skin-snip data, Remme et al. (1990) provide a model based estimate of the average longevity of onchocerciasis infection and its variability by fitting observed trends in prevalence and intensity of infection during vector control. However, these authors note that their estimate represents only one possible quantification and that a detailed sensitivity analysis is required to determine the complete range of parameter values which are consistent with observations.

The present paper provides such a detailed analysis. The comprehensive stochastic

Correspondence address: A.P. Plaisier, Department of Public Health and Social Medicine, Medical Faculty, Erasmus University Rotterdam, P.O. Box 1738, 3000 DR Rotterdam, The Netherlands. 
model for onchocerciasis transmission and control, ONCHOSIM (Plaisier et al., 1990), is used to systematically test different parameter quantifications of the worm lifespan distribution using longitudinal skin-snip data collected in selected reference villages during 13-14 years of vector control operations in the area of the Onchocerciasis Control Programme in West Africa (OCP). The changes in microfilarial load are not only determined by the parasite lifespan. Therefore, other aspects are explicitly taken into account, including the pre-control endemicity level and infection heterogeneity among the inhabitants of the reference villages.

The aim of the analysis reported here is to arrive at a plausible range of values for the average reproductive lifespan of the West African Savanna strain of the $O$. volvulus parasite, and for the age at which the large majority of the worms have reached the end of their reproductive period. Especially the latter, which we will characterize by the age at which $95 \%$ of the adult worms have ceased reproduction, is an important determinant of the period during which vector control must be continued to minimize the risk of recrudescence of onchocerciasis.

\section{Materials and Methods}

\section{Epidemiological surveys and reference villages}

Epidemiological surveys have been undertaken at regular intervals of 3-4 years in a large number of villages in the OCP in order to monitor the epidemiological impact of vector control (WHO, 1987a). In all surveys two skin-snips were taken from each person. These snips were incubated in distilled water for $30 \mathrm{~min}$, examined by microscope for the presence and density of $O$. volvulus microfilariae (mf), and the number of mf per snip was recorded for each individual at each survey (Prost and Prod'hon, 1978). From this extensive epidemiological data-set, four villages have been selected as reference for the analysis. Table 1 lists some demographic and epidemiological characteristics of these villages, which are all located in Burkina Faso in the core area of the OCP and which have been under vector control for at least 13 years. The selection of the villages was such that they fitted into the general pattern for the epidemiological trends observed during the vector control period (see Remme et al., 1990). All 4 villages were hyperendemic (prevalence of $\mathrm{mf}>60 \%$, Prost et al., 1979) but they were chosen to cover a fairly wide range for the intensity of infection as measured by the Community Microfilarial Load (CMFL), i.e. the geometric mean $\mathrm{mf} / \mathrm{snip}$ for all individuals older than 20 years (Remme et al., 1986). At least four surveys were done in each of the villages.

\section{The model}

Simulations have been carried out with the stochastic computer simulation model ONCHOSIM (Plaisier et al., 1990). An important characteristic of ONCHOSIM is the use of the technique of microsimulation. The life-histories of individual persons and their individual inhabitant male and female worms are simulated. Together, the persons constitute a simulated village population. In the model, the reproductive lifespan is defined as the period between inoculation of the infective larva and the end of the fecundity period of the adult worm. This includes the pre-mature period 
in which the worm cannot yet reproduce. The end of fecundity may be due to factors such as disease or death of the worm. The requirement of regular mating is explicitly considered in the model and a temporary cessation of mf-production when no mating takes place is not considered as the end of fecundity. The average reproductive lifespan is taken to be equal for male and female worms. In the remainder of the analysis, all causes leading to the end of the fecund period are simply referred to as 'death', while 'reproductive lifespan' is abbreviated to 'lifespan'. The variability in the lifespan in considered to be solely the result of the worm's genetic variability and does not depend on intrinsic host factors. The lifespan is described by a probability distribution of the Weibull type. The Weibull distribution is widely used in reliability models for lifetime of devices and for survival analyses (see Kalbfleisch and Prentice, 1980). It is a generalization of the one parameter exponential distribution which is often used for describing lifetimes in infectious disease models (see Anderson and May, 1985). The Weibull has a 'shape' parameter in addition to the mean for describing the variability of the lifespan. We will frequently use the 95 th percentile, i.e. the age at which $95 \%$ of the parasites have died. This measure depends both on the mean and the shape (see Appendix for further details).

Apart from the lifespan, the model enables a detailed description of a number of other aspects of the worm life-history. Fecundity starts after a pre-mature period of one year on average (Duke, 1980; Prost, 1980). Following this period, mf production only takes place when worms mate (Schulz-Key and Karam, 1986). In accordance with several observations, the mf-output of fertilized female parasites is modelled to decrease with age after a few years of optimal productivity (Albiez, 1985; Karam et al., 1987). The appendix gives details about modelling worm mating and fecundity. Newly produced $\mathrm{mf}$ are assumed to live for 1.5 years. In the current quantification of the model one fertilized female worm at peak fecundity corresponds with an average of $5 \mathrm{mf} / \mathrm{snip}$ (this value is arrived at in an analysis which will be reported elsewhere).

The model takes account of the fact that the exposure to (new) infections in the pre-control situation is unequally distributed in the human population. This exposure heterogeneity, which acts as a major determinant of differences in skin-snip counts between persons, results from differences between sexes (females are on average $20 \%$ less exposed than males), differences between ages (beyond the age of 20 years the maximum exposure is reached), and individual variation which is, among others, related to attractivity to the flies and behavioural factors. This individual variation can take different values for different village populations.

A detailed description of the model and of the parameter quantifications is available on request.

\section{Estimation procedure}

The validity of different quantifications of the model parameters are tested by determining the goodness-of-fit of simulated skin-snip data to the observed skin-snip results. For this purpose, both the observed and simulated skin-snip counts are summarized in a frequency distribution with geometric class boundaries: $0,0.5,1,2$, ..., $256 \mathrm{mf} / \mathrm{snip}$. The analysis is restricted to cohorts of persons who have been snipped at each survey and who were older than 20 years at the first survey. The restriction to adults follows previous arguments that it is only for this age group that 
one may expect a stable age-structure of the initial parasite population and, therefore, regular epidemiological trends during the control period (Remme et al., 1986). Furthermore, only the first survey and the surveys after 10 years of control are used for the sensivity analysis because in particular the differences between early and late surveys are sensitive to variations in the quantification of the parasite lifespan parameters.

The goodness of fit is characterized by the $P$ value of a $\chi^{2}$ test. Each comparison of a simulated and observed skin-snip distribution renders one $P$ value. Since we are interested in the goodness-of-fit with all relevant surveys, the results for separate surveys for a given reference village can be combined into one overall $P$ value. Finally, $P$ values for different villages can be combined into an overall measure for the goodness-of-fit. The procedure for combining $P$ values is described in the appendix.

Because of the difficulties involved in assessing the correct number of degrees of freedom in a model in which a number of parameters are estimated simultaneously from data from different sources, the $P$ value resulting from the $\chi^{2}$ test should not be used as a formal criterion for accepting or rejecting assumptions on the lifespan. Instead, it will be used to identify regions with good and poor fitting parameter values. Therefore, we will use the term 'score' instead of $P$ value in the remainder. Goodness of fit scores have been determined for many combinations of values for the mean lifespan (between 7 and 12 years, with steps of 1 year) and for the 95 th percentile (between 9 and 20 years).

Table 1 strongly suggests that the pre-control endemicity of the villages (as measured by the CMFL of the first survey) is different and, hence, that the average vector biting density before the start of control must have been different. However, average biting rates are unknown. Therefore, each quantification of the lifespan parameters is tested for a range of values for the pre-control biting rate such that the observed endemicity levels fall within the range of simulated endemicity levels. As mentioned before, the reference villages also differ in their degree of exposure heterogeneity. Testing of lifespan quantifications for a village is therefore always done for the village specific best fitting values of the biting rate and exposure heterogeneity.

\section{Results}

\section{Lifespan parameters}

Fig. 1 shows for each of the villages the goodness-of-fit score as a function of the two lifespan parameters. The 95th percentile is plotted on the horizontal axis and the values for the mean lifespan are represented by different lines. The scores are plotted on the vertical axis using a reversed log-scale, which implies that values in the upper part of the graph represent poor fitting model quantifications, whereas values close to the horizontal axis represent good fitting, plausible quantifications. For each village, the four values for the mean lifespan are shown which give the best fit.

A straightforward conclusion from Fig. 1 is that only a limited range for the mean lifespan and the 95th percentile gives a good fit with the trends observed in the villages. At both sides of an optimal range for the two lifespan parameters, the 
TABLE 1

Characteristics of the four villages that are used for testing the assumptions on the parasite lifespan

\begin{tabular}{llllll}
\hline Village & River basin & $\begin{array}{l}\text { Initial } \\
\text { census } \\
\text { population }\end{array}$ & $\begin{array}{l}\text { Size } \\
\text { of study } \\
\text { cohort }\end{array}$ & $\begin{array}{l}\text { CMFL at } \\
\text { first survey } \\
\text { (mf/snip) }\end{array}$ & $\begin{array}{l}\text { Timing of surveys (yrs, since } \\
\text { start of control) }\end{array}$ \\
\hline Tiercoura & Leraba & 160 & 31 & 53 & $0.8,5.0,7.0,10.0,12.0,14.0$ \\
Folonzo & Comó & 285 & 48 & 30 & $0.8,4.9,7.0,10.0,12.0,14.0$ \\
Sarba-Baforo & Bougouriba & 600 & 76 & 17 & $1.4,8.9,11.0,13.3$ \\
Loaba & White Volta & 270 & 19 & 41 & $1.8,5.1,10.1,13.1$ \\
\hline
\end{tabular}

Folonzo

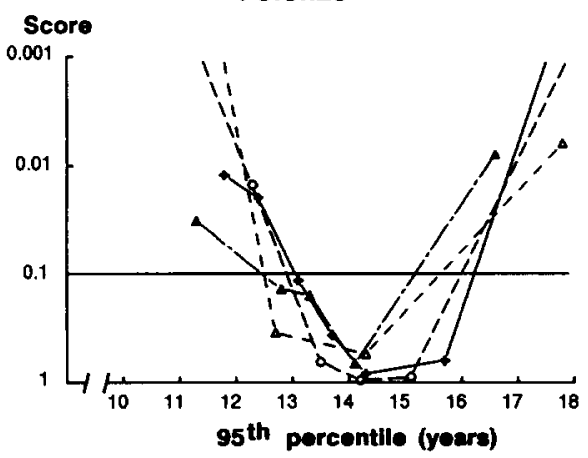

Sarba-Baforo

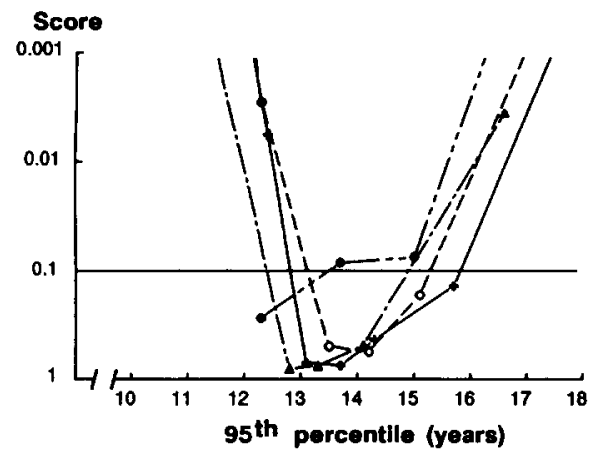

Loaba

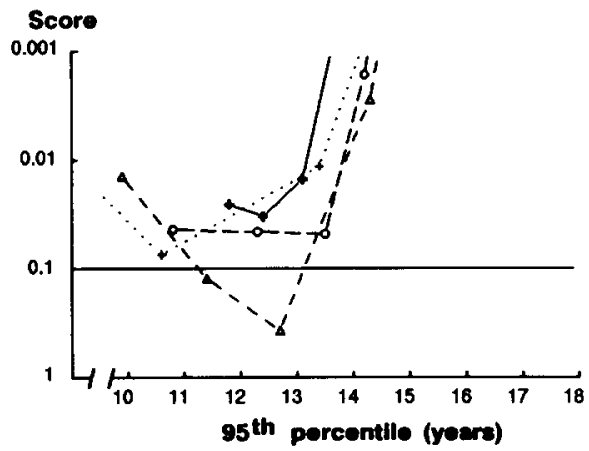

Tlercoura

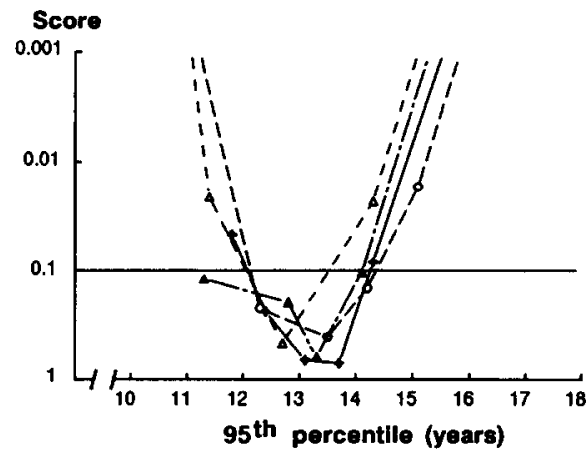

Fig. 1. Goodness of fit scores between simulation results and observed skin-snip count distributions in the 4 reference villages for different values of the mean lifespan (years) and the age at which $95 \%$ of all parasites have died ( 95 th percentile, years). Results are plotted on a reversed log-scale, i.e. lower values indicate a better fit. For each village, only the four best fitting values of the mean lifespan are plotted. As a result, a mean lifespan of 12 years is only included for Sarba-Baforo, and 7 years only for Loaba. Symbols: Mean lifespan (years) $+\cdot \cdot+=7 ; \Delta-\triangle=8 ; O-O=9 ;+-+=10 ; \Delta-\Delta=11 ; 0-\bullet=12$.

goodness-of-fit gets worse. The only exception is the mean lifespan of 12 years fitted to data of Sarba-Baforo, which shows a good score even when there is very little variation (95th percentile of 12.3). Both the width and the position of the optimal range is not identical for the four villages, as should be the case, if only because of random sampling fluctuations. The data for Folonzo and Sarba-Baforo allow for a 
fairly wide range for the 95th percentile while a more limited range is obtained from Tiercoura and Loaba. Except for Loaba, at least three values for the mean lifespan give rise to very high scores (close to the horizontal axis). In Loaba, only one value for the mean lifespan ( 8 years) gives a reasonable fit with the data. In this village there is also a preference for low values of the 95 th percentile. In contrast, Folonzo shows a preference for higher values of the 95 th percentile.

A more regular picture, with a more restricted range for the 95 th percentile is obtained when the results of the villages are combined. This is shown in Fig. 2. Loaba is not included in this combination. It is so different from the other villages, that no acceptable over-all fit could be obtained for all 4 villages combined.

A summary of the results in Table 2, together with Figs. 1 and 2, shows that the best fit is obtained for a mean lifespan of 9 to 11 years and a 95th percentile of 13 to 15 years.

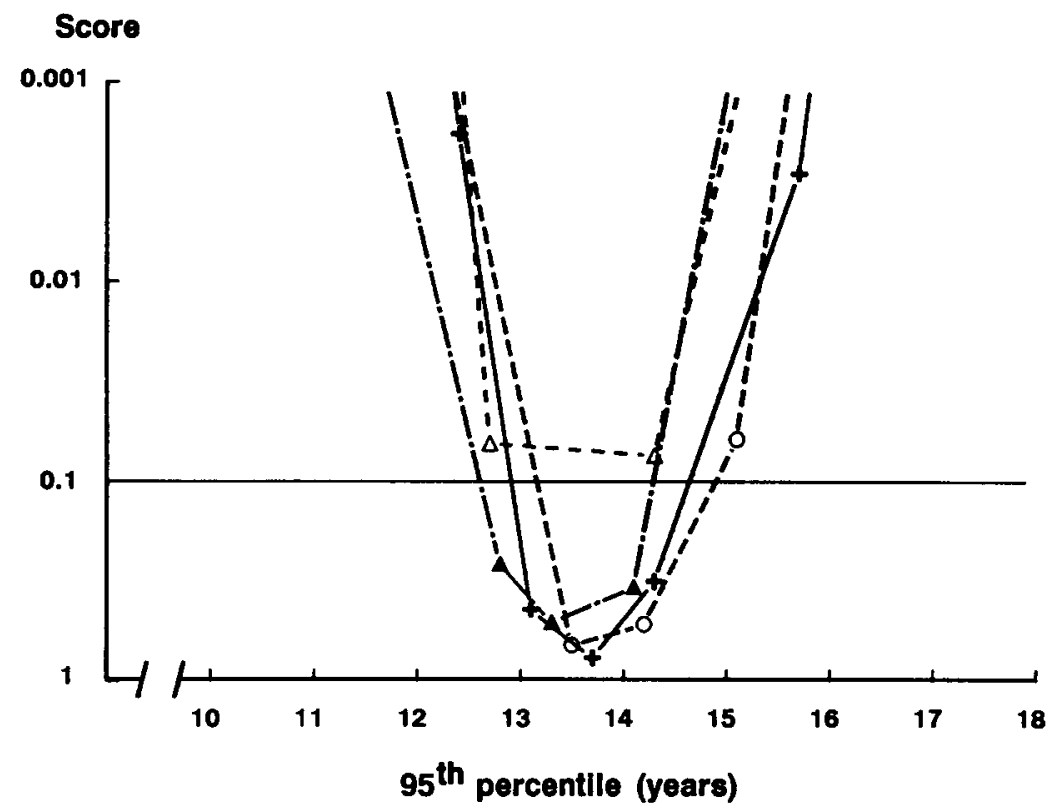

Fig. 2. Goodness of fit score as a function of the mean lifespan and the 95 th percentile for the combined test results of Folonzo, Sarba-Baforo, and Tiercoura. See also Fig. 1. (For explanation of symbols see Fig. 1.)

TABLE 2

Ranges for the mean lifespan and the 95th percentile with a goodness of fit score $>0.1$ for the 4 reference villages and for the combination of Folonzo, Sarba-Baforo, and Tiercoura

\begin{tabular}{lll}
\hline Village & Mean lifespan & 95 th percentile \\
\hline Tiercoura & $8-11$ & $11-14.5$ \\
Folonzo & $7-11$ & $12.5-16$ \\
Sarba-Baforo & $8-12$ & $12.5-16$ \\
Loaba & 8 & $11-13$ \\
Combination (excl. Loaba) & $9-11$ & $12.5-15$ \\
\hline
\end{tabular}




\section{Example of simulated $m f$ distributions}

In addition to the goodness-of-fit test, it is important to have a visual check of the appropriateness of the estimates for the description of a given data set. As an example, in Fig. 3 for Folonzo the observed mf distributions from three surveys are shown together with simulated distributions. In this simulation, the mean lifespan is 10 years and a 95 th percentile 13.7 years (which is a plausible quantification given the results of Figs. 1 and 2). Fig. 3 shows that the change in the $\mathrm{mf}$ distribution is simulated quite well.

\section{Simulated trends in prevalence of $\mathrm{mf}$ and in $C M F L$}

Skin-snip distributions are usually summarized to mf-prevalence and CMFL, the level of onchocerciasis infection. Parameter quantifications with a good fit appear to provide also a close agreement between observed and simulated trends in these indices, see Fig. 4. The exception is again the Loaba data, which can only be fitted with a lower value for both the mean lifespan and the 95 th percentile. This is clear from the overestimation of the infection prevalence.

\section{Discussion}

Reliable estimates of the parasite reproductive lifespan are not only important for the quantitative understanding of endemic onchocerciasis, but also for the prediction of the epidemiological impact of vector control and the determination of the required duration of vector control (see a.o. Remme et al., 1986, 1990; Habbema et al., 1990). Several previous attempts at estimation of the lifespan have been made, though often on the basis of limited data.

Roberts et al. (1967) reported on onchocerciasis infection levels after elimination of the vector from several isolated foci in Kenya. Cross-sectional surveys showed still live adult worms present in nodules and alive $\mathrm{mf}$ in the skin 11 years after interruption of transmission, but no more $\mathrm{mf}$ in the skin 18 years after vector elimination. These authors postulated, therefore, that $O$. volvulus loses its reproductive potential after a lifespan of 16 years or possibly earlier. Though this study did not explicitly address the question of variability, it is fair to assume that their postulate refers to an upper limit for the reproductive lifespan.

A direct estimate of the duration of onchocerciasis infection is provided by Duke (1980) who recorded the intervals between inoculation of infective larvae in chimpanzees, the appearance of $\mathrm{mf}$ in the skin and the final disappearance of skin $\mathrm{mf}$. Based on an experiment in a single chimpanzee, he found for the savanna strain of $O$. volvulus an interval of less than 6 years between the last inoculation and the complete disappearance of $\mathrm{mf}$ from the skin. Though it cannot be excluded that the last inoculum was ineffective, this duration (which includes both the adult parasite lifespan and the microfilarial lifespan) is extremely short and does not correspond with other estimates and with the trends observed in the OCP.

Karam et al. (1987) present an analysis of epidemiological data obtained during the first 7 to 8 years of the OCP. Based on longitudinal changes in skin mf loads and in differences in the adult worm composition as compared to non-controlled areas, 

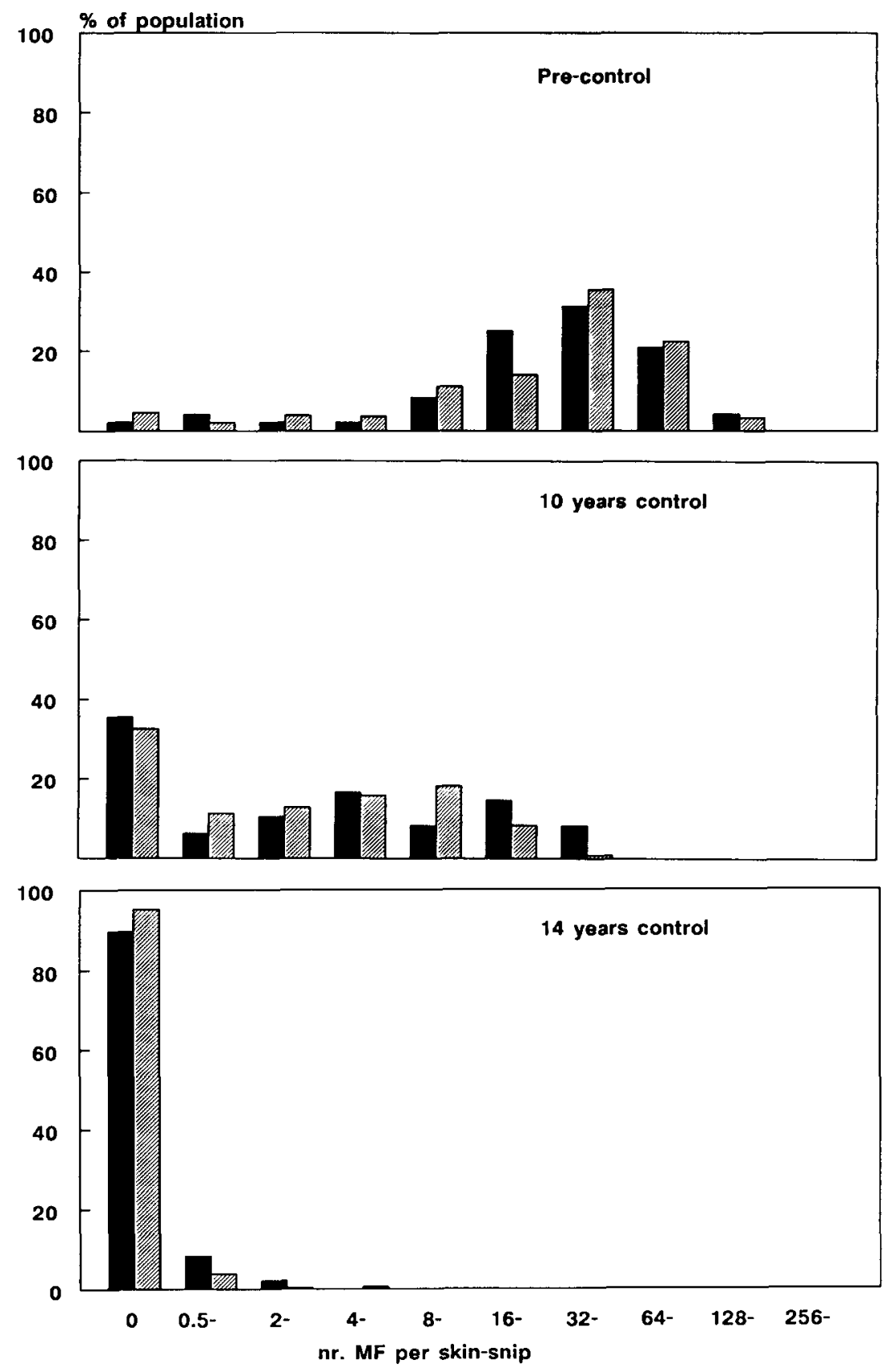

Fig. 3. Folonzo: Simulated and observed trend in skin-snip count distribution. In the simulation the mean lifespan is 10 years and the 95 th percentile is 13.7 years. $=$ observed; $=$ simulated.

they postulate a breakdown of the worm population 11 to 12 years after the beginning of control. Furthermore, they demonstrate that the mf productivity was significantly reduced in aged worms.

The question of variability in the reproductive lifespan was first addressed in the 
Folonzo

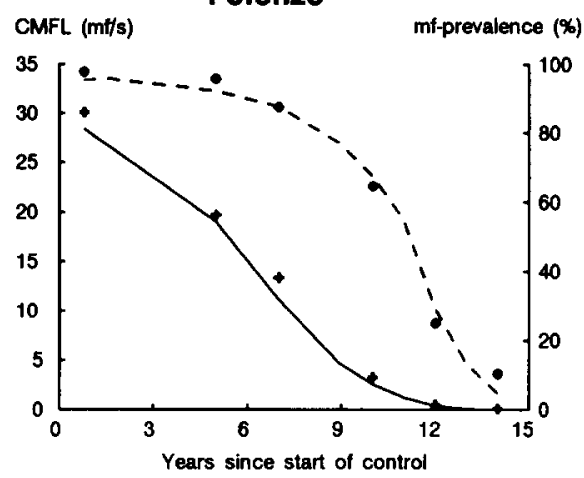

Sarba-Batoro

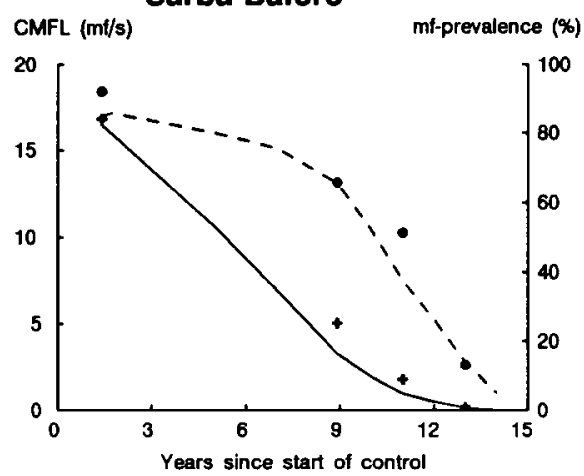

Loaba

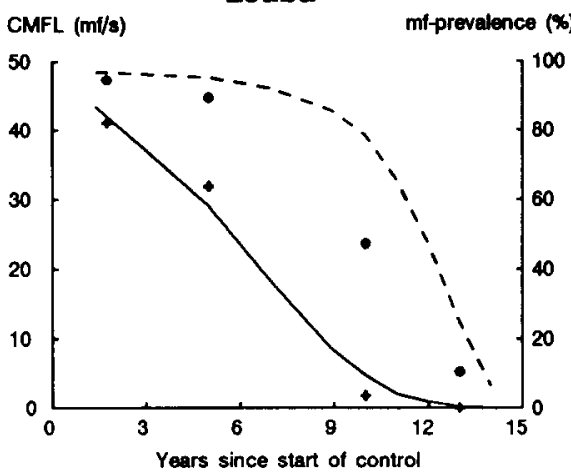

Tiercoura

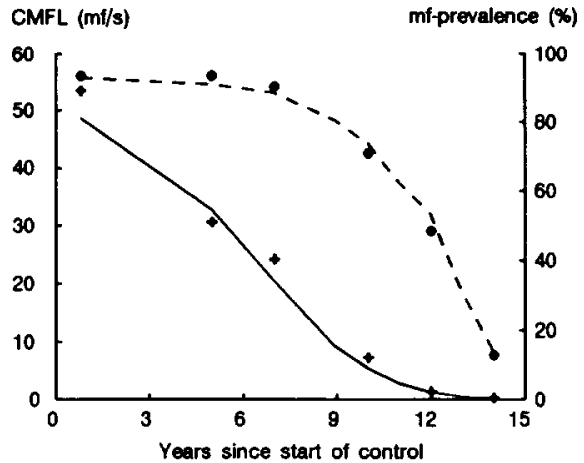

Fig. 4. Simulated and observed trends in CMFL and $\mathrm{mf}$-prevalence in the four reference villages. The simulations use a mean lifespan of 10 years and a 95 th percentale of 13.7 years. Symbols: $+=\mathrm{CMFL}$ observed; $-=$ CMFL simulated; $-\mathrm{mf}$ prevalence observed; $---=\mathrm{mf}$ prevalence simulated.

study by Remme et al. (1990). Following the introduction of a simple force-ofinfection model, which led to a preliminary estimate of 11 years for the average duration of infection (Remme et al., 1986), they developed a more sophisticated hostparasite model which actually was a predecessor of ONCHOSIM. The host-parasite model was quantified using longitudinal skin-snip data for the first 8-9 years of vector control in the OCP and this resulted in an estimate for the mean longevity of infection (including $\mathrm{mf}$ survival) of 10.4 years and a variability which was such that $95 \%$ of the longevities were less than 15 years. The model allowed the prediction of trends in the prevalence of skin mf and in the CMFL for different levels of endemicity and it was shown that the predicted trends gave a good description of those observed in 55 villages during 12-14 years of control. However, no formal estimation procedures were followed in this study and no attempts were made to investigate other quantifications.

The distinguishing feature of the present analysis is the systematic search for plausible quantifications of the major parameters which determine the reproductive life-history of $O$. volvulus. The model used, ONCHOSIM, takes account of all aspects that ultimately determine the mean duration of onchocerciasis infection and its variability. Several model simplifications have been considered in order to arrive at a 
more simple and tractable estimation procedure. A first simplification would be to assume a (single-parameter) exponential distribution for the lifespan (see, among others, Anderson and May, 1985). However, this assumption is not compatible with the trends and is especially in conflict with the accelerated decline in mf-prevalence during the late years of control (10 to 14 years, see Fig. 4). Also the restricted range of the 95th percentile of the lifespan distribution, which can be concluded from Figs. 1 and 2, demonstrates the inappropriateness of the exponential distribution which has still a 95 th percentile of 21 years at the very low mean of 7 years.

By systematically varying model assumptions it appeared that only with a considerable degree of age- and sex-independent exposure variation between individuals a good fit is obtained between simulated and observed skin-snip distributions. A simple model which disregards this personal variation, could be fitted to trends in aggregated measures like CMFL and $\mathrm{mf}$ prevalence, but is not suitable for describing the more detailed data that we have available.

Two rather detailed assumptions that are not necessary for obtaining a fit of the skin-snip data are the age-dependent fecundity and the requirement of regular mating for the female worms. The assumption of a constant fecundity level - though conflicting with nodulectomy results (see Karam et al., 1987) - will lower the estimates of the lifespan. When female worms can produce $\mathrm{mf}$ even in the absence of male worms, the residual worm population in the final years of control will be much more productive and this must also be compensated by a shorter lifespan. The inclusion of these refinements which cannot be tested against skin-snip data is justified because of the (biological) face validity of the model. This is important in view of the role of ONCHOSIM in prospective evaluation of control measures.

Other parameters that influence the mean duration of infection include the $\mathrm{mf}$ production per worm and the lifespan of $\mathrm{mf}$. Variations in these parameters are expected to influence the estimate of the worm lifespan. Alternative quantifications of the $\mathrm{mf}$ production level have been tested by using the same goodness-of-fit procedure as reported in this paper. Lower values for the lifespan (especially the 95th percentile) result when a lower mf production per worm, and thus a higher worm load, is assumed. The opposite applies to a high per-worm productivity. However, these alternative quantifications for the $\mathrm{mf}$ production will affect the lifespan estimates with at most 0.5 year.

Another parameter which may still alter our estimates is the microfilarial lifespan, which we have taken equal to 1.5 years. We tested the influence of other values between 0.5 and 2 years, which is the widest range range compatible with the observations of Duke (1968). Intuitively, one could expect that a change in the lifespan of the microfilariae will be compensated by exactly the opposite change in the lifespan of the macrofilariae. Thus, a mf lifespan of 0.5 years instead of 1.5 years would add 1 year to the worm lifespan. However, because of a reduced mf-production of old worms, this mechanism does not apply exactly. It was found that values of 0.5 or 2 years for the $\mathrm{mf}$ lifespan will not change our estimates of the worm reproductive lifespan with more than 0.5 year.

Different alternative values for the length of the prepatent period have been tested. This revealed that, in the range of 0.5 to 1.5 years, these alternative values do not influence the estimate of the lifespan.

The goodness-of-fit results suggest some systematic differences between the lifespan estimates which were obtained with the longitudinal data for each of the four villages. 
Some of these differences may reflect discrepancies between actual transmission patterns and the model assumptions of stable pre-control transmission levels and complete interruption of transmission during the vector control period. Circumstantial evidence suggests that the actual transmission pattern around the start of control may have been different in at least three villages and we will therefore discuss each village in some detail.

The lowest estimate for the lifespan was obtained with the data for the village of Loaba. This village is located along the White Volta River, much more to the east than the other three villages. It has been noted previously that the decline in infection levels was much faster in the north-east of the original OCP area. This has been attributed to a major reduction or even interruption of transmission during the last years of the pre-control period when there was hardly any local vector breeding because of severe drought and when there was no more vector invasion from the south-west where vector control started one to two years earlier than in the east (Karam et al., 1987; Remme et al., 1990). If local transmission was indeed absent for one or two years before the start of control, than our mean lifespan estimate of 8 years for Loaba, is an underestimate.

The highest estimates for the mean lifespan were obtained with the data for SarbaBaforo. This village is located along the River Bougouriba, which was during the first few years of vector control subject to reinvasion by infective flies from outside the Programme area (Garms et al., 1979). This initial reinvasion may have been responsible for a significant incidence of (super)infection and this has been given as the explanation for the different epidemiological trends in the first line villages along this river, where the CMFL started to show the predicted linear decline only after a delay of a few years (WHO, 1987b). Sarba-Baforo is not a first line village and no significant delay in the decline of the CMFL has been observed previously. However, it is possible that the detailed analysis presented here has detected some minor impact of the reinvasion phenomenon.

Extensive entomological evaluation data are available for a fly catching point near the village of Folonzo where vector control resulted in an immediate reduction in fly biting rates. Nevertheless, the Annual Transmission Potential, an entomological index of $O$. volvulus transmission (Walsh et al., 1978), was during the first three years of control equal to 274,50 and 107 infective larvae per years respectively before falling to levels close to zero during subsequent years. Annual Transmission Potentials in the range of 100 to 200 infective larvae per year are usually taken as evidence of a low level of active transmission (Thylefors et al., 1978), but it should be noted that the fly population during the first three years of control at Folonzo consisted nearly exclusively of reinvading flies which stay close to the riverine vegetation and have less chance of man-fly contact (Garms et al., 1979). One may speculate, therefore, that there have been occasional new infections during the first three years of control which explain the higher estimate for the 95 th percentile but which were too few to affect the estimate for the mean lifespan.

At the fourth village of Tiercoura there has never been a catching point, but available evidence from the two nearest catching points at a distance of 8 and 17 kilometers respectively suggest that complete control of the vector population has been achieved since the start of the larviciding operations.

Combining the results of our analysis, the above background information about the four reference villages, and the results of previous studies, we conclude that the 
mean reproductive lifespan of the savanna strain of $O$. volvulus lies between 9 and 11 years, and that $95 \%$ of the parasites reach the end of reproduction before the age of 13 to 14 years.

\section{Appendix}

Worm mating and age dependent fecundity in the model

According to Schulz-Key and Karam (1986) and Schulz-Key (1990), a reproductive cycle of a female $O$. volvulus worm takes about 3 months, and hence for ongoing microfilarial production, insemination must take place at 3 monthly intervals. The mating probability is taken to be equal to the ratio of male to female worms, with a value of 1.0 when there are more male than female worms. By means of random number generation on the basis of this probability, it is determined for each female worm that needs insemination; i.e. for which the last insemination took place at least 3 months ago - whether (re-)insemination takes place indeed. If not, then mfproduction ceases immediately. Each month, all uninseminated female worms have a new chance to mate. It is obvious that in the case of very low (residual) worm loads, the worm mating probabilities in the human host will be reduced and will vary considerably from host to host.

Apart from mating, the mf-output is also dependent on the age of the worms. We tested several models for the age-specific fecundity. In the simulations of the present analysis, female worms have their maximum fecundity during the first 5 years of patency. So, with an average pre-patent period of 1 year, this maximum level of fecundity ends at the age of 6 years. Thereafter, fecundity reduces linearly, and becomes zero at the (practically unattainable) patent age of 20 years.

Finally, it is assumed that, due to genetic variability and due to the differential distance to the snipping site, inseminated female worms of the same age can make a different contribution to the skin-snip count. The latter variability is described by an exponential distribution function.

\section{Combining test results}

Test results for several surveys or for several villages are combined as follows:

1. The $P$ value obtained from a goodness of fit test is under the null hypothesis uniformly distributed on $[0,1]$. Hence, $-\ln (P)$ is exponentially distributed with a mean of 1 .

2. In combining the $P$ values of $n$ tests $\left(P_{1}, P_{2}, \ldots, P_{n}\right)$, we use the fact that the sum of $n$ independent exponentially distributed random variables follows an $n$-Erlang distribution.

Thus,

$$
x=\sum_{i=1}^{n}-\ln \left(P_{i}\right)
$$

follows the $n$-Erlang distribution: 


$$
F(x)=\mathrm{e}^{-x} \sum_{j=0}^{n-1} \frac{x^{j}}{j !}
$$

3. $F(x)$ is the goodness-of-fit score for the combination of $n$ tests.

\section{Lifespan distribution}

The lifespan is described by a Weibull distribution, using an offset of 4 years, which implies that no parasite will reach the end of the reproductive period before the age of 4 years.

One of the plausible distributions is characterized by a mean lifespan of 10 years and a 95th percentile of 13.7 years. This distribution can be described as follows (to be read as the probability to be dead before the age of $x$ ):

$$
F(x)= \begin{cases}0 & \text { if } 0<x<4 \\ 1-\mathrm{e}^{-(x / \beta) \alpha} & \text { if } x>4\end{cases}
$$

with: $\alpha=2.97$ and $\beta=6.72$.

Other percentiles of this distribution: 80 th $=11.9 ; 90$ th $=12.9 ; 99$ th $=15.2$.

\section{Acknowledgements}

We would like to thank Dr. E.M. Samba, Director OCP, for his support of our work. The analysis reported here was carried out on the basis of a Technical Service Agreement, number $08 / 181 / 85$, provided by the World Health Organisation, on behalf of the Onchocerciasis Control Programme (OCP).

\section{References}

Albiez, E.J. (1985) Calcification in adult Onchocerca volvulus. Trop. Med. Parasit. 36, 180-181.

Anderson, R.M. and May, R.M. (1985) Helminth infections of humans: mathematical models, population dynamics and control. Adv. Parasitol. 24, 1-101.

Duke, B.O.L. (1968) The effects of drugs on Onchocerca volvulus I. Methods of assessment, population dynamics of the parasite and the effects of diethylcarbamazine. Bull. Wld. Hith. Org. 39, 137-146.

Duke, B.O.L. (1980) Observations on Onchocerca volvulus in experimentally infected chimpanzees. Tropenmed. Parasit. 31, 41-54.

Garms, R.. Walsh, J.F. and Davies, J.B. (1979) Studies on the reinvasion of the Onchocerciasis Control Programme in the Volta River Basin by Simulium damnosum s.l. with emphasis on the south-western areas. Tropenmed. Parasit. 30, 345-362.

Habbema, J.D.F., Plaisier, A.P., van Oortmarssen, G.J. and Remme, J. (1990) Prospective evaluation of onchocerciasis control strategies. Acta Leiden. 59, 387-398.

Kalbfleisch, J.D. and Prentice, R.L. (1980) The Statistical Analysis of Failure Time Data, Wiley, New York.

Karam. M., Schulz-Key, H. and Remme, J. (1987) Population dynamics of Onchocerca volvulus after 7 to 8 years of vector control in West Africa. Acta Trop. 44, 445-457.

Plaisier, A.P., van Oortmarssen, G.J., Habbema, J.D.F., Remme, J. and Alley, E.S. (1990) ONCHOSIM: A model and computer simulation program for the transmission and control of onchocerciasis. Comp. Meth. Prog. Biomed. 31, 43-56. 
Prost, A. and Prod'hon, J. (1978) Le diagnostique parasitologique de l'onchocercose, revue critique des méthodes en usage. Med. Trop. 38, 519-532.

Prost, A., Hervouet, J.P. and Thylefors, B. (1979) Les niveaux d'endémicité dans l'onchocercose. Bull. Wld. Hlth. Org. 57, 655-662.

Prost, A. (1980) Latence parasitaire dans l'onchocercose. Bull. Wld. Hlth. Org. 58, 923-925.

Remme, J., Ba, O., Dadzie, K.Y. and Karam, M. (1986) A force-of-infection model for onchocerciasis and its application in the epidemiological evaluation of the Onchocerciasis Control Programme in the Volta River basin area. Bull. Wld. Hlth. Org. 64, 667-681.

Remme, J., de Sole, G. and van Oortmarssen, G.J. (1990) The predicted and observed decline in onchocerciasis infection during 14 years of successful Simulium control in West Africa with reference to the reproductive lifespan of Onchocerca volvulus. Bull. Wld. Hlth. Org., in press.

Roberts, J.M.D., Neumann, E., Göckel, C.W. and Highton, R.B. (1967) Onchocerciasis in Kenya 9, 11 and 18 years after elimination of the vector, Bull. Wld. Hlth. Org. 37, 195-212.

Schulz-Key, H. and Karam, M. (1986) Periodic Reproduction of Onchocerca volvulus, Parasitol. Today 2, 284-286.

Schulz-Key, H. (1990) Observations on the reproductive biology of Onchocerca volvulus. Acta Leiden. 59. $27-43$.

Thylefors, B., Philippon, B. and Prost, A. (1978) Transmission potentials of Onchocerca volvulus and the associated intensity of infection of onchocerciasis in a Sudan-savanna area. Tropenmed. Parasit. 29, 346-354.

Walsh, J.F., Davies, J.B., Le Berre, R. and Garms, R. (1978) Standardization of criteria for assessing the effect of Simulium control in onchocerciasis control programmes. Trans. R. Soc. Trop. Med. Hyg. 72, 675-676.

WHO. (1987a) Expert committee on onchocerciasis, 3rd report, World Heaith Organization, Geneva.

WHO. (1987b) Twelve years of onchocerciasis control in Western Africa. World Health Statistics Annual 1987, 19-22. 